Memorias del VII Encuentro Nacional de Experiencias en la Enseñanza de la Biología y la Educación Ambiental y II Congreso Nacional de Investigación en la Enseñanza de la Biología

\title{
APORTE DEL DISEÑO DE UNIDADES DIDÁCTICAS A LA FORMACIÓN DE FUTUROS DOCENTES DE CIENCIAS NATURALES Y EDUCACIÓN AMBIENTAL DE LA UNIVERSIDAD SURCOLOMBIANA
}

\author{
Jeniffer Rivas Aviléz ${ }^{1}$
}

\section{Elías Francisco Amórtegui Cedeño²}

\section{Resumen}

En este documento presentamos resultados preliminares acerca de la sistematización del diseño de unidades didácticas para la enseñanza de las ciencias naturales. El grupo de trabajo consistió en 23 futuros docentes con edades que oscilaron entre los 20 y 25 años, pertenecientes a estratos socioeconómicos 1 y 2 que cursaron el espacio académico de Didáctica I durante el segundo semestre del 2012, en el Programa de Licenciatura en Educación Básica con Énfasis en Ciencias Naturales y Educación Ambiental de la Universidad Surcolombiana (Neiva, Colombia). La metodología estuvo enmarcada en un enfoque cualitativo, empleando el análisis de contenido y la sistematización de la primera entrega del diseño de las unidades didácticas.

\section{Abstract}

This paper presents preliminary results on the study of future teachers' conceptions of the undergraduate program in Basic Education with Emphasis in Natural Sciences from the University Environmental Education Surcolombiana-USCO(Neiva-Colombia). The study was conducted from a qualitative perspective, based on content analysis and data collection based on participant observation, the workshop and the questionnaire. The results show a marked tendency toward ideas related to a traditional didactic approach in relation to the theory and practice, practice field image and evaluation of this type of teaching strategies.

Palabras Clave: unidad didáctica, conocimiento didáctico, enseñanza, aprendizaje, conocimiento profesional

Keywords: didactic unit, didactic knowledge, teaching, learning, professional knowledge.

\footnotetext{
${ }^{1}$ Estudiante de séptimo semestre de Licenciatura en Educación Básica con Énfasis en Ciencias Naturales y Educación Ambiental. Universidad Surcolombiana. jenyra90@gmail.com

2 Docente de Planta Tiempo Completo. Programa de Licenciatura en Educación Básica con Énfasis en Ciencias Naturales y Educación Ambiental. Universidad Surcolombiana. Magister en Educación. elias.amortequi@usco.edu.co
} 
Bio-grafía Escritos sobre la Biología y su Enseñanza.

Edición Extra-Ordinaria. ISSN 2027-1034 P.p 762 - 770

Memorias del VII Encuentro Nacional de Experiencias en la Enseñanza de la Biología y la Educación Ambiental y II Congreso Nacional de Investigación en la Enseñanza de la Biología

\section{Introducción}

En los últimos años la profesión docente se ha subestimado social y epistemológicamente al considerarse que para enseñar basta con tener dominio del conocimiento disciplinar a profundidad, dejando de lado el hecho de que como profesionales de la educación, los docentes poseen un conocimiento particular que además de incluir lo disciplinar (en este caso ya sea lo biológico, lo físico o lo químico), incluye aspectos didácticos y pedagógicos, lo que les posibilita enseñar adecuadamente diferente a como lo pueden realizar los profesionales de otras áreas (Valbuena, 2007); debido a lo anterior, actualmente se están realizando diversas investigaciones que llevan a mejorar esta situación; como resultado de dichos estudios se plantea la importancia de investigar en el ámbito de la formación docente los procesos que promueven la construcción de dicho conocimiento y su vinculación en la formación inicial de maestros (Tardif, 2004).

Para el caso de profesores de ciencias naturales en educación básica, los estudios desde la perspectiva del Conocimiento Profesional también son escasos tanto a nivel colombiano como internacional (Martínez y Molina, 2009). En términos globales Abell (2008) plantea que después de 20 años de investigación sobre el Conocimiento de los Profesores y particularmente sobre el Pedagogical Content Knowledge, aún queda una gran cantidad de investigaciones por realizar.

La revisión de antecedentes muestra que a nivel internacional existen algunos estudios que se han realizado con profesores de una sola disciplina, ya sea Biología, Química o Física, sin embargo las investigaciones con profesores de Ciencias Naturales y Educación Ambiental son escasos, resaltando así la importancia, relevancia y pertinencia de los presentes hallazgos preliminares.

\section{Metodología}

El estudio se llevó a cabo desde un enfoque cualitativo, empleando el método de análisis de contenido en el proceso de sistematización. El grupo objeto de estudio consistió en 23 estudiantes que oscilan 20 y 25 años, pertenecientes a estratos socio-económicos 1 y 2 que cursaron el espacio académico de Didáctica I durante el segundo semestre del 2012, en el Programa de Licenciatura en Educación Básica con Énfasis en Ciencias Naturales y Educación Ambiental de la Universidad Surcolombiana (Neiva, Colombia). El procedimiento para el análisis de contenido para esta investigación tuvo en cuenta los siguientes aspectos: primero se identificaron las fuentes de información, luego se ubicaron las unidades de información (UI) de cada fuente, las cuales corresponden a afirmaciones textuales en dichos documentos que ofrecieron información con sentido y significado propio para la investigación, posteriormente se les asignó un código. Luego las UI se 
Bio-grafía Escritos sobre La Biologia y su Enseñanza.

Edición Extra-Ordinaria. ISSN 2027-1034 P.p 762 - 770

Memorias del VII Encuentro Nacional de Experiencias en la Enseñanza de la Biología y la Educación Ambiental y II Congreso Nacional de Investigación en la Enseñanza de la Biología

sistematizaron de acuerdo a cuatro categorías (Enseñanza, Contenidos de enseñanza, Aprendizaje y Formación docente), las cuales corresponden en términos generales a la Didáctica de las Ciencias Experimentales. Las unidades didácticas corresponden a tres grupos de trabajo, grupo 1 enseñanza de la respiración, grupo 2 enseñanza de la reproducción y grupo 3 enseñanzas de la excreción.

\section{Resultados y discusión}

A continuación se presentan los hallazgos en cada una de las categorías, en algunos casos se ilustra con declaraciones textuales de los futuros profesores de Ciencias Naturales y Educación Ambiental (se destaca la información relevante subrayando).

\section{Contenidos de enseñanza}

En la primera entrega, la mayoría de los estudiantes establecieron como elementos en el diseño de la unidad didáctica aspectos relacionados exclusivamente desde la perspectiva tradicional de la enseñanza de las ciencias naturales en donde priman los contenidos conceptuales; sin embargo, algunos pocos estudiantes explicitaron la importancia de tener en cuenta elementos procedimentales y actitudinales.

G1.E1.1. "[Haciendo referencia a los objetivos de aprendizaje en la unidad didáctica]

- Mostrar un alto grado de sensibilidad y respeto por el medio ambiente y todas las formas de vida.

- Trabajar en equipo, con el fin de potenciar la reflexión, el contraste de opiniones y la obtención de conclusiones, para así poder desarrollar capacidades de cooperación entre los estudiantes."

G2.E1.1. "[Haciendo referencia a los objetivos de aprendizaje en la unidad didáctica]

- Realizar trabajos, talleres individuales y grupales que ayuden en la profundización de conceptos e ideas planteadas.

- Realizar foros y trabajos de grupo, en el cual se den a conocer las ideas y conocimientos que tengan los estudiantes sobre la reproducción y su relación en la vida cotidiana."

G3.E1.1. "[Haciendo referencia a los objetivos de aprendizaje en la unidad didáctica] Comparar e identificar los mecanismos utilizados en los seres vivos para eliminar desechos dañinos para él." 
Bio-grafía Escritos sobre la Biología y su Enseñanza.

Edición Extra-Ordinaria. ISSN 2027-1034 P.p 762 - 770

Memorias del VII Encuentro Nacional de Experiencias en la Enseñanza de la

Biología y la Educación Ambiental y II Congreso Nacional de Investigación en la Enseñanza de la Biología

Lo anterior está relacionado en su mayoría con la perspectiva academicista de la enseñanza de las ciencias, en la cual priman los contenidos conceptuales y por otro lado en menor proporción una perspectiva significativa-sistémica en la cual se propende por la interrelacionen de manera integral los contenidos conceptuales, procedimentales y actitudinales (Valbuena, 2007). Esto, con el propósito de que le den sentido al mundo de lo vivo y de su entorno, y que, además, puedan aplicar dichos conocimientos de una manera significativa y crítica a su vida cotidiana mediante la valoración, conservación y aprovechamiento sostenible de los recursos y que esto les permita asumir posiciones críticas de orden bio-ético ante situaciones de la vida contemporánea, más aún cuando en la ciudad de Neiva existen problemáticas ambientales, tales como: el abandono y la falta de conciencia de la comunidad en el manejo de los recursos hídricos como es el caso de la Laguna del Curibano, el mal manejo de los desechos sólidos, la tala de árboles, la poca arborización, la contaminación auditiva, entre otros.

Por otra parte, la situación anterior está relacionada con la estructura curricular del programa ya quela formación en Didáctica de las Ciencias Naturales corresponde exclusivamente al sexto y séptimo semestre y además a que las prácticas pedagógicas se llevan a cabo únicamente durante el último año de formación posterior a los espacios académicos de Didáctica I y Didáctica II.

\section{Aspectos formativos docentes}

Los estudiantes en la primera entrega, resaltaban la importancia de cinco aportes formativos docentes, algunos de ellos mencionando una sola tendencia y otros compartían algunas de ellas, las tendencias mencionadas fueron: activismo, secuencia de la enseñanza, estrategias, herramientas y aspectos profesionales; la primera hace referencia a que en la unidad didáctica los futuros maestros deben hacer explicito las actividades que permitan a los alumnos además de mejorar sus relaciones con sus compañeros, construir significados activamente, modificando, sustituyendo o ampliando ideas y conceptos existentes; en la segunda tendencia se referencia por parte de dos grupos de futuros maestros la secuencia de la enseñanza, distribuyendo el contenido y precisando como se desarrollará el esquema conceptual de la unidad didáctica; en la tercera tendencia un grupo de futuros docentes habla de las estrategias y las nuevas alternativas para el buen desarrollo de una unidad didáctica; dos grupos en la cuarta tendencia mencionan las herramientas necesarias para el desarrollo de la unidad didáctica y la última tendencia un grupo menciona un enfoque profesional para que la unidad didáctica fortalezca capacidades profesionales e intelectuales.

G1.E1.2 [Haciendo referencia a los aspectos formativos docentes en la unidad didáctica] "Utilizar el diseño de una unidad didáctica para fortalecer capacidades profesionales $e$ intelectuales con el propósito de ofrecer al estudiante una 
Bio-grafía Escritos sobre la Biología y su Enseñanza.

Edición Extra-Ordinaria. ISSN 2027-1034 P.p 762 - 770

Memorias del VII Encuentro Nacional de Experiencias en la Enseñanza de la Biología y la Educación Ambiental y II Congreso Nacional de Investigación en la Enseñanza de la Biología

formación educativa, humana y social a través de la unidad didáctica."

G2.E1.2[Haciendo referencia a los aspectos formativos docentes en la unidad didáctica] "Dar a conocer estrategias y nuevas alternativas para el buen desarrollo de una unidad didáctica"

G3.E1.2[Haciendo referencia a los aspectos formativos docentes en la unidad didáctica] "Diseñar la secuencia de enseñanza, distribuyendo el contenido y precisando como se desarrollara el esquema conceptual de la unidad didáctica"

En primera medida es de resaltar que estas afirmaciones de los futuros maestros trascienden la idea predominante de que basta con que el profesor domine académicamente elconocimiento disciplinar que enseña, para que sea un buen profesor. Sin embargo es de destacar que la mayoría de profesores en formación conciben la unidad didáctica exclusivamente desde una perspectiva instrumentalo activista, en la que se enfocan en las actividades y formas de enseñar contenidos biológicos. Lo ideal sería lograr que los futuros docentes de Ciencias Naturales y Educación Ambiental conciban la unidad didáctica como un ejercicio formativo que les permita la integración de contenidos escolares, estrategias metodológicas, finalidades de enseñanza, ideas de los alumnos sobre las ciencias naturales, dificultades de enseñanza-aprendizaje y evaluación de los aprendizajes, en aras de que reconozcan que el diseño de la unidad didáctica permite identificar el conocimiento que se construye en el aula de clase y que este difiere del conocimiento producido por los científicos, pues se trata de un conocimiento escolar epistemológicamente diferenciado, que ha de producirse didácticamente a partir de las transformaciones e integraciones de diferentes saberes y conocimientos que confluyen en la escuela (Valbuena et al, 2009).

Teniendo en cuenta lo anterior, es importante que en la formación de maestros se trabaje porque éstos se reconozcan como sujetos de conocimientos propios de su profesión. La formación del profesorado debe partir de un enfoque reflexivo, en el que se analicen las prácticas profesionales, las tareas y los conocimientos de los maestros (Tardif, 2004).Barnett y Hodson (2001) consideran que no basta con que el docente adquiera competencias para aplicar los referentes teóricos sino requiere, además, poseer un conocimiento tal que lo capacite para tomar decisiones en contextos educativos particulares, por lo que en la formación inicial del profesorado es importante que se propicien espacios para que los futuros docentes vivencien los ambientes escolares y reflexionen sobre las idiosincrasias particulares de los profesores en ejercicio y su incidencia en el ejercicio profesional. 
Bio-grafía Escritos sobre la Biología y su Enseñanza.

Edición Extra-Ordinaria. ISSN 2027-1034 P.p 762 - 770

Memorias del VII Encuentro Nacional de Experiencias en la Enseñanza de la Biología y la Educación Ambiental y II Congreso Nacional de Investigación en la Enseñanza de la Biología

\section{Enseñanza}

En la primera entrega de la elaboración de la unidad didáctica, los futuros docentes hacen énfasis en tres grandes concepcionesacerca delproceso de enseñanza; dos grupos resaltan la importancia de las ideas previas, uno de esos grupos habla sobre la necesidad de romper con la educación tradicional y un último un grupo menciona la gran relevancia de ver el contexto de la población de la unidad didáctica con el fin de llevar la teoría a la práctica.

G1.E1.3 [Haciendo referencia a los aspectos de la enseñanza en la unidad didáctica] "La utilización de las ideas previas de los estudiantes, el enfoque de problemas acordes al contexto (Ciencia, Tecnología y sociedad), el aprendizaje basado en problemas, es una metodología activa donde los estudiantes participan en la constante búsqueda y construcción del conocimiento...."

G2.E1.3 [Haciendo referencia a los aspectos de la enseñanza en la unidad didáctica] "El problema consiste en que la mayoría de los docentes se quedan trabajando de una forma muy tradicional y poco usual hoy día para los estudiantes."

G2.E1.5 [Haciendo referencia al contexto de la problemática de la unidad didáctica] "Antes de iniciar la unidad didáctica se debe indagar sobre los conocimientos previos que tienen las alumnas de los temas relacionados con esta, se debe preguntar que saben de los reinos existentes en la naturaleza, que tipos de reproducción conocen, las diferencias de reproducción entre los reinos, para así saber las fortalezas y debilidades que tienen las estudiantes; y para saber que metodología emplear y que tanto profundizar en el desarrollo de dicha unidad didáctica."

G3.E1.3 [Haciendo referencia a los aspectos de la enseñanza en la unidad didáctica] "Hablando de la realidad de nuestra práctica docente se da como un momento que vivimos en el presente, en este texto plantea la necesidad del estudio de ese presente, en el análisis de la realidad se intenta reconocer opciones derivadas de un proyecto a hacer posible, no de probar hipótesis, no se pretende aplicar una estructura teórica sino descubrir aquella que contribuya a esclarecer lo objetivamente posible." 
Bio-grafía Escritos sobre La Biologia y su Enseñanza.

Edición Extra-Ordinaria. ISSN 2027-1034 P.p 762 - 770

Memorias del VII Encuentro Nacional de Experiencias en la Enseñanza de la Biología y la Educación Ambiental y II Congreso Nacional de Investigación en la Enseñanza de la Biología

Lo anterior es fundamental dado que de acuerdo a Pozo y Gómez (1998), es relevante el tratamiento de las concepciones de los alumnos como el punto de partida para la acumulación de conflictos cognitivos que le permitan el aprendizaje de las ciencias, en donde es clave que los escolares activen sus conocimientos o teorías previas permitiéndole al profesor conocer las diferentes concepciones alternativas de sus alumnos; en este sentido, las ideas de los futuros maestros están relacionados con un modelo didáctico constructivista plantea que en un modelo constructivista en el que es fundamental el paralelo entre la construcción del conocimiento científico y el conocimiento de los alumnos, de esta forma la enseñanza-aprendizaje de las ciencias parte de una fase de exploración de ideas previas, una reestructuración de conocimientos, una introducción de ideas nuevas y la aplicación de estas a nuevos contextos Jiménez (2000).

Desde esta perspectiva, la enseñanza se encamina a lograr una evolución en los educandos y se priorizan los procesos cognitivos orientados a los cambios conceptual, procedimental y actitudinal. Así pues, más que enseñar contenidos específicos, se busca el cambio de las estructuras cognitivas de los alumnos (Valbuena, 2007).

Es fundamental entonces que los futuros maestros de Ciencias Naturales y Educación Ambiental, reconozcan que las estrategias y actividades de enseñanza que se implementan, en este caso las planteadas en la unidad didáctica, además de tener en cuenta las concepciones de los alumnos, su nivel de desarrollo cognitivo, sus intereses, tengan en cuenta sus experiencias y su contexto sociocultural (Amórtegui, 2011). .

\section{Aprendizaje}

Con relación a esta categoría, cabe destacar que tan sólo el grupo 3 reconoce algunos elementos relacionados con aquellos sujetos que están aprendiendo y en ese sentido argumentan que el proceso de aprendizaje de los estudiantes está relacionado con aspectos como lo actitudinal, lo cual debe ser acompañado por el maestro.

G3.E1.6 [Haciendo referencia a los aspectos de la enseñanza en la unidad didáctica] "tenemos que ayudar al niño comenzando por observar detalladamente los problemas o actitudes del alumno que le impidan llevar a cabo su progreso de una manera natural".

Con base en lo anterior puede establecerse que los futuros maestros sobrepasan la idea de los alumnos aprenden con el mero hecho de percibir el objeto de 
Bio-grafía Escritos sobre la Biologia y su Enseñanza.

Edición Extra-Ordinaria. ISSN 2027-1034 P.p 762 - 770

Memorias del VII Encuentro Nacional de Experiencias en la Enseñanza de la Biología y la Educación Ambiental y II Congreso Nacional de Investigación en la Enseñanza de la Biología

aprendizaje, o solamente a partir de la explicación del profesor (siempre y cuando se esté atento), dicho aprendizaje ocurre de una manera directa, y no median procesos entre lo nuevo y lo que posee el alumno; en este sentido los estudiantes se acercan a visiones más complejas sobre el aprendizaje de las Ciencias Naturales y Educación Ambiental, en las cuales el aprendizaje es un proceso metacognitivo, idiosincrásico e intrínseco y por lo tanto no basta con que el profesor conozca las ideas de los alumnos, sino que es menester que los estudiantes sean conscientes de ellas, de sus obstáculos y de su progresión, en donde es fundamental un aprendizaje enriquecido con la motivación, preferiblemente intrínseca, la constante autorregulación, la autorreflexión, la autoevaluación, la autosuperación de los obstáculos, el protagonismo y la apropiación de los objetivos de formación (Valbuena, 2007).

\section{Conclusiones}

La unidad didáctica diseñada por los futuros maestros permitió explicitar en las respectivas entregas sus ideas acerca de enseñanza, aprendizaje, contenidos de enseñanza y la formación docente. Con relación a la enseñanza, los docentes en formación conciben la importancia del uso de las ideas previas y la necesidad de romper con la educación tradicional; frente a los contenidos de enseñanza los estudiantes establecieron como elementos en el diseño de la unidad didáctica aspectos relacionados exclusivamente desde la perspectiva tradicional de la enseñanza de las ciencias naturales en donde priman los contenidos conceptuales; sin embargo, algunos pocos estudiantes explicitaron la importancia de tener en cuenta elementos procedimentales y actitudinales; con relación al aprendizaje tan sólo un grupo dio cuenta de la importancia de la motivación de los estudiantes como un elemento que favorece el aprendizaje de las Ciencias Naturales y la Educación Ambiental y en cuanto a los aspectos formativos, establecen la importancia de explicitar las actividades que permitan a los alumnos además de mejorar sus relaciones con sus compañeros, construir significados activamente, además la unidad didáctica permitió secuencias la enseñanza a través de estrategias y tan solo un grupo de trabajo identificó elementos sobre el fortalecimiento de capacidades profesionales e intelectuales.

\section{Bibliografía}

- Abell, S. (2008a). Twenty Yaers Later: Does Pedagogical Content Knowledge remain a useful idea? International Journal of ScienceEducation. 30 (10), 1405-1416.

- Amórtegui, E (2011). Concepciones sobre prácticas de campo y su relación con el conocimiento profesional del profesor, de futuros docentes de Biología de la Universidad Pedagógica Nacional. Título para optar al título de Magíster 
Bio-grafía Escritos sobre la Biologia y su Enseñanza.

Edición Extra-Ordinaria. ISSN 2027-1034 P.p 762 - 770

Memorias del VII Encuentro Nacional de Experiencias en la Enseñanza de la

Biología y la Educación Ambiental y II Congreso Nacional de Investigación en la Enseñanza de la Biología

en Educación de la Universidad Pedagógica Nacional. Departamento de Posgrados: Bogotá.

- Barne-tt, J. y D. Hodson (2001), "Pedagogical context knowledge: toward a fuller understanding of what good science- teachers know", Science Teacher Education, núm. 85, p-p-. 426-453.

- Jiménez, M (2000). Modelos didácticos. En PERALES J y CAÑAL P (Coord) Didáctica de las Ciencias Experimentales. Editorial Marfil Alcoy: España.

- Martínez, c \& Molina, A (2009). El conocimiento profesional de los profesores de ciencias de primaria sobre el conocimiento escolar en el distrito capital: un problema de investigación. Memorias I congreso Nacional de investigación en educación en ciencias y tecnología, 2009, Junio 22 a 26.

- Pozo, J y Gómez, M (1998). Aprender y enseñar ciencia. Del conocimiento cotidiano al conocimiento científico. Madrid: Ediciones Morata.

- Tardif, M. (2004). Los saberes del docente y su desarrollo profesional. Traducción de Pablo Manzano. Madrid: Narcea.

- Valbuena, E. (2007). El Conocimiento Didáctico del Contenido Biológico. Estudio de las concepciones disciplinares y didácticas de futuros docentes de la Universidad Pedagógica Nacional (Colombia). Tesis para optar al título de Doctor en Didáctica de las Ciencias Experimentales. Universidad Complutense de Madrid.

- Valbuena, Édgar; Gutiérrez, Alice; Correa, Mónica; y Amórtegui, Elías. (2009). Procesos formativos que favorecen la construcción del conocimiento profesional del profesor en futuros docentes de Biología. Revista Colombiana de Educación. No 56. Pp 156-179. 